\title{
SAÚDE BUCAL E QUALIDADE DE VIDA NO PACIENTE PÓS-RADIOTERAPIA DE CÂNCER DE CABEÇA E PESCOÇO
}

\author{
Laura Cavalcanti de Oliveira* \\ Debora Foger Teixeira* \\ Cássia Maria Fischer Rubira ${ }^{*+*}$ \\ Paulo Sérgio da Silva Santos ${ }^{* * * *}$
}

\begin{abstract}
RESUMO: A radioterapia no paciente com câncer de cabeça e pescoço pode resultar em muitas consequências negativas, como a hipossalivação. Sem a proteção da saliva há um risco aumentado de cáries e doenças periodontais que podem causar a perda dentária e consequentemente influenciam no impacto da saúde bucal na qualidade de vida. 0 objetivo desse estudo foi avaliar o impacto da saúde bucal na qualidade de vida dos pacientes pós-radioterapia de câncer de cabeça e pescoço por meio das avaliações de fluxo salivar e edentulismo. A amostra foi composta por 19 indivíduos com idade a partir de 18 anos e diagnóstico de câncer de cabeça e pescoço. A radioterapia do tipo Intensidade Modulada (IMRT) foi 0 tratamento mais realizado 16 $(68,4 \%)$. Referente ao tempo pós-radioterapia, nove $(47,4 \%)$ haviam terminado 0 tratamento há um ano. Quanto ao impacto da saúde bucal na qualidade de vida, 15 (79,9\%) apresentaram algum impacto. 0 maior número de pacientes, sete (36,8 \%), não usava algum tipo de prótese e nove $(47,4 \%)$ necessitavam de uma combinação de reabilitações. Encontraram-se 15 (78,9\%) pacientes com fluxo salivar classificado como muito baixo e quatro $(21,1 \%)$ mostraram melhor fluxo salivar após cinco anos da radioterapia. 0 teste de Coeficiente de Correlação de Pearson mostrou correlação entre a necessidade de prótese e a idade $(p=0,006 ; r=0,4734)$ e a necessidade de prótese e o fluxo salivar dos pacientes $(p=0,03 ; r=0,3672)$. As complicações da radioterapia em cabeça e pescoço acarretam uma série de consequências físicas e psicológicas. 0 tempo após a radioterapia parece influenciar, em alguns casos, a melhora do fluxo salivar e em menos de um ano de tratamento não há impacto da saúde bucal na qualidade de vida. São necessários estudos longitudinais com essa população para a verificação dos efeitos da radioterapia ao longo dos anos.
\end{abstract}

PALAVRAS-CHAVE: Neoplasias de cabeça e pescoço; Radioterapia; Saúde bucal; Qualidade de vida.

\section{MOUTH HEALTH AND LIFE QUALITY IN THE PATIENT HEAD AND NECK CANCER AFTER RADIOTHERAPY}

\begin{abstract}
Radiotherapy in a patient with head and neck cancer may produce several negative consequences, such as hypo-salivation. Due to lack of protection by saliva, increase in the number of caries and other periodontal diseases is a risky problem which may cause teeth loss and may impact life quality through mouth health. The impact of mouth health on life quality of post-radiotherapy patients with head and neck cancer is evaluated by evaluating saliva flow and toothlessness. Sample comprised 19 individuals, over 18 years, diagnosed with head and neck cancer. Modulated Intensity Radiotherapy featured in 16 (68.4\%) patients, of whom nine (47.4\%) ended treatment one year before. Fifteen patients (79.9\%) experienced mouth health impacting their life quality. Seven patients $(36.8 \%)$ did not wear any prosthesis and nine (47.4\%) required a combination of rehabilitations. Fifteen $(78.9 \%)$ patients had low saliva flow and four $(21.1 \%)$ had better saliva flow after five years of radiotherapy. Pearson's Coefficient of $\mathrm{C} 0-$ relation revealed co-relationship between the need for prosthesis and age $(\mathrm{p}=0.006$; $r=0.4734)$ and the need of prosthesis and saliva flow $(p=0.03 ; r=0.3672)$. Radiotherapy complications on head and neck bring several physical and psychological issues. In some cases, the post-radiotherapy period improves saliva flow, but there is no impact of mouth therapy on life quality in a less-than-one-year treatment. Longitudinal studies with such populations are required to investigate deeply the effect of radiotherapy throughout the years. KEYWORDS: Head and neck cancer; Radiotherapy; Mouth health; Life quality.

\footnotetext{
Discente. Universidade de São Paulo (FOB USP), Brasil. E-mail: lauracavalcanti459@gmail.com

** Doutorando no departamento de estomatologia/radiologia/patologia e cirurgia da Faculdade de Odontologia de Bauru, Brasil.

${ }^{*}$ Docente doutora da Faculdade de Odontologia de Bauru (FOB USP) no departamento de estomatologia/radiologia/patologia e cirurgia, Brasil.

${ }^{* * * t}$ Docente doutor da Faculdade de Odontologia de Bauru (FOB USP) no departamento de estomatologia/radiologia/patologia e cirurgia, Brasil.
} 


\section{INTRODUÇÃo}

As neoplasias malignas tornaram-se um problema de saúde pública mundial, causando grande impacto individual e social (CORREIA, 2013). As neoplasias de cabeça e pescoço representam cerca de $10 \%$ de todas as neoplasias malignas e englobam várias áreas anatômicas. Em média $40 \%$ dos casos ocorre na cavidade oral, $25 \%$ na laringe, $15 \%$ na faringe, $7 \%$ nas glândulas salivares e $1 \%$ nos órgãos restantes (CORREIA, 2013; MARQUES 2015).

Os tratamentos disponíveis para as neoplasias de cabeça e pescoço são a cirurgia, a radioterapia e a quimioterapia. A escolha do tratamento depende da localização e do tipo histológico do tumor, bem como o grau de comprometimento do órgão em questão e as condições físicas do indivíduo, a fim de eliminar 0 tumor com o mínimo de sequelas possíveis (BROWWER et al., 2015; CHEN et al., 2011; CORREIA, 2013).

A radioterapia é uma modalidade de tratamento que utiliza energia ionizante eletromagnética ou corpuscular com 0 objetivo de destruir as células tumorais. Entretanto, 0 tratamento ionizante não é seletivo e atinge também as células saudáveis (CORREIA, 2013; MARQUES, 2015). A unidade que mede as doses de radiação é denominada Gray (Gy). Frequentemente, os indivíduos com neoplasias de cabeça e pescoço recebem uma dose entre 50 e 70 Gy, que é aplicada de maneira fracionada, dividida por um período de cinco a sete semanas, uma vez ao dia, cinco dias na semana, sendo que a dose diária gira em torno de 2 Gy (MARQUES, 2015, ROLIM et al., 2011). Os efeitos colaterais, geralmente, podem aparecer a partir de doses baixas de $10 \mathrm{~Gy}$, ocorrendo de forma aguda ao tratamento radioterápico, como disfagia, mucosite, sangramento, odinofagia, candidíase, periodontopatias, xerostomia, disgeusia, rouquidão, emagrecimento e alterações de pele, incluindo eritema, descamação, dor, ardência, formação de bolhas e em alguns casos necrose tecidual, e/ou de forma tardia, meses e até mesmo anos após o término do tratamento como trismo, fibrose de tecido subcutâneo, queda de cabelo, cárie de radiação, ulceração de pele ou mucosa, alterações auditivas, oftalmológicas e hormonais, necrose de cartilagens, dormência dos membros superiores, mielite cervical, osteorradionecrose, diminuição da contração faríngea, redução do reflexo de deglutição, entre outros (CHEN et al., 2011, CORREIA, 2013, HUTCHESON et al., 2012; MARQUES, 2015; THARIAT et al., 2012; TOLENTINO et al., 2011).

A saliva tem papel fundamental na manutenção de condições fisiológicas bucais consideradas normais, assim como contribui nas propriedades antimicrobianas, na gustação, fala, formação do bolo alimentar, e auxilia no processo de retenção protética (PEDRAZAS et al., 2007). Por essa razão, todas suas funções acarretam em melhor qualidade de vida do paciente, uma vez que diminui alterações bucais como doença periodontal, lesões de cárie, candidíase, disfagia decorrentes da hipossalivação, assim como fornece maior estabilidade protética, principalmente em processos mastigatórios (ALMEIDA e KOWALSKI, 2010; BRAGA, 2006; CABRERA et al., 2007; FEIO e SAPETA, 2005). 0 paciente com câncer de cabeça e pescoço enfrenta, além de problemas com sua saúde sistêmica, mudanças mentais e bucais que influenciam sua vida social e familiar (JEMAL et al.,2009). A análise de todos os efeitos colaterais da radioterapia, tanto na saúde bucal como no impacto da saúde bucal na qualidade de vida, leva o profissional a tomar cuidados devidos com todas as alterações presentes, bem como as que surgirão (HADDAD e SHIN, 2008). A vista disso é de extrema importância a avaliação e diagnóstico das complicações bucais oriundas do tratamento radioterápico, bem como 0 impacto negativo dessas complicações na qualidade de vida do paciente, objetivando melhores estratégias de tratamento e, consequentemente, diminuindo o impacto na qualidade de vida. Portanto, o objetivo desse estudo foi avaliar 0 fluxo salivar, edentulismo e o impacto da saúde bucal na qualidade de vida de pacientes com câncer de cabeça e pescoço após o tratamento radioterápico. 


\section{METODOLOGIA}

0 projeto foi aprovado pelo Comitê de Ética em Pesquisa em Seres Humanos da Faculdade de Odontologia de Bauru - Universidade de São Paulo. Número do parecer: 2.020.434.

Foram convidados para participar do estudo todos os indivíduos maiores de 18 anos atendidos no Centro de Pesquisa Clínica da Faculdade de Odontologia de Bauru - USP no período de agosto de 2016 a agosto de 2017, com diagnóstico de câncer de cabeça e pescoço que realizaram o tratamento radioterápico, excluindo aqueles que apresentavam história prévia de déficit neurológico. A amostra foi então composta por 19 indivíduos.

A avaliação do fluxo salivar foi realizada por meio da sialometria de repouso, que é a análise quantitativa e qualitativa da saliva, em que o paciente a despreza em um copo de boca larga de $5 \mathrm{~mL}$ sem estímulo mecânico, com duração de 5 minutos. Obtém-se então uma média de $\mathrm{mL} / \mathrm{min}$ dividindo os $\mathrm{mL}$ coletados por 5 . Valores obtidos inferiores a $0,25 \mathrm{~mL} /$ min demonstraram que o paciente possui baixo fluxo salivar ou hipossalivação. (TARZIA, 2004). Para o uso e a necessidade de prótese foi utilizado o instrumento da Organização Mundial da Saúde (AZEVED0, 2014). Os participantes foram avaliados na cadeira odontológica por um profissional cirurgião-dentista devidamente calibrado.

0 impacto da saúde bucal na qualidade de vida foi avaliado por meio do instrumento OHIP-14, 0 qual é composto por 14 questões divididas em sete dimensões: limitação funcional, dor física, desconforto psicológico, incapacidade física, incapacidade psicológica, incapacidade social e desvantagem social. As respostas para 0 questionário foram dadas por meio de uma escala de Likert, em que: $0=$ nunca; $1=$ raramente; $2=$ às vezes; $3=$ frequentemente e $4=$ sempre (FOGER, 2015). 0 instrumento foi aplicado ao participante por meio de uma entrevista, por um profissional devidamente calibrado.

\section{RESULTADOS}

A idade média dos 19 participantes foi de 58 anos (33 a 71), sendo 16 (84,2 \%) do sexo masculino e $15,8 \%$ do sexo feminino. 0 diagnóstico microscópico foi de carcinoma espinocelular em 13 $(68,4 \%)$ pacientes, carcinoma epidermoide $2(10,5 \%)$, carcinoma mucoepidermoide $1(5,2 \%)$, carcinoma anexial 1 (5,2\%), tumor de corda vocal $1(5,2 \%)$ e linfoma maligno não Hodgkin 1 (5,2\%). 0 carcinoma espinocelular na língua foi a doença de base mais prevalente - oito - $(42,1 \%)$, acometendo também laringe - três - (15,7\%), seio piriforme - um - $(5,2 \%)$, nasofaringe - um - $(5,2 \%)$ e palato mole - um - $(5,2 \%)$. A radioterapia do tipo Intensidade Modulada (IMRT) foi o tratamento mais realizado - 16 - $(68,4 \%)$, outros três $(31,6 \%)$ realizaram cobaltoterapia. Referente ao tempo pós-radioterapia, nove $(47,4 \%)$ haviam terminado 0 tratamento há um ano.

Quanto ao impacto da saúde bucal na qualidade de vida, 15 (79,9\%) apresentaram algum impacto. 0 maior número de pacientes - sete - $(36,8$ \%) não usava nenhum tipo de prótese e nove $(47,4 \%)$ necessitavam de uma combinação de reabilitações. Encontraram-se 15 (78,9\%) pacientes com fluxo salivar classificado como muito baixo e quatro $(21,1$ \%) mostraram melhor fluxo salivar após cinco anos da radioterapia (Tabela 1). 0 teste de Coeficiente de Correlação de Pearson (Tabela 2) mostrou correlação entre a necessidade de prótese e a idade $(p=0,006$; $r=0,4734)$ e a necessidade de prótese e o fluxo salivar dos pacientes ( $p=0,03 ; r=0,3672)$. 
Tabela 1. Dados relacionados à radioterapia, qualidade de vida e índices de saúde bucal

(Continua na outra coluna)

\begin{tabular}{|c|c|c|c|}
\hline Tipos de Radioterapia & N (\%) & Tipos de Radioterapia & N (\%) \\
\hline Radioterapia de Intensidade Modulada & $13(68,4 \%)$ & \multicolumn{2}{|l|}{ OHIP-14 } \\
\hline Cobaltoterapia & $3(15,8 \%)$ & Forte & $4(21,1 \%)$ \\
\hline Números de Sessões & & Médio & $4(21,1 \%)$ \\
\hline 39 & $2(10,5 \%)$ & Fraco & $11(57,9 \%)$ \\
\hline 33 & $4(21,1 \%)$ & \multicolumn{2}{|l|}{ Uso de Prótese } \\
\hline 35 & $5(26,3 \%)$ & Não usa & $7(36,8 \%)$ \\
\hline 30 & $2(10,5 \%)$ & Usa uma ponte fixa & $1(5,3 \%)$ \\
\hline 27 & $1(5,3 \%)$ & Usa mais que uma ponte fixa & $2(10,5 \%)$ \\
\hline 28 & $1(5,3 \%)$ & Usa uma ponte parcial removível & $3(15,8 \%)$ \\
\hline 29 & $1(5,3 \%)$ & Usa uma ou mais ponte fixa / uma ou mais & $1(5,3 \%)$ \\
\hline Tempo Pós Radioterapia & & Usa uma ponte total & $5(26,3 \%)$ \\
\hline Até 1 ano & $7(36,8 \%)$ & \multicolumn{2}{|l|}{ Necessidade do uso de prótese } \\
\hline De 1 a 5 anos & $5(26,3 \%)$ & Não necessita & $4(21,1 \%)$ \\
\hline De 5 a 10 anos & $4(21,1 \%)$ & Necessita de prótese fixa ou prótese & $1(5,3 \%)$ \\
\hline De 10 a 15 anos & $1(5,3 \%)$ & Necessita de prótese fixa ou prótese & $4(21,1 \%)$ \\
\hline De 10 a 20 anos & 0 & Necessita de combinação de próteses & $9(47,4 \%)$ \\
\hline \multirow[t]{3}{*}{ Mais de 20 anos } & 0 & \multicolumn{2}{|l|}{ Fluxo salivar } \\
\hline & & Muito baixo & $15(78,9 \%)$ \\
\hline & & Melhor resultado após cinco anos & $4(21,1 \%)$ \\
\hline
\end{tabular}

Fonte: Dados da pesquisa

Tabela 1. Resultado da análise de correlação de Pearson entre as variáveis estudadas

\begin{tabular}{|c|c|c|c|c|c|c|c|}
\hline & Idade & \begin{tabular}{|c} 
Tempo pós- \\
rádio
\end{tabular} & OHIP-14 & $\begin{array}{c}\text { Fluxo } \\
\text { salivar }\end{array}$ & $\begin{array}{l}\text { Uso de } \\
\text { prótese }\end{array}$ & Dose total & $\begin{array}{c}\text { Fluxo } \\
\text { estimulado }\end{array}$ \\
\hline Idade & $\begin{array}{l}r=1,0000 \\
p=---\end{array}$ & $\begin{array}{l}r=-, 0019 \\
p=, 992\end{array}$ & $\begin{array}{l}r=, 0668 \\
p=, 717\end{array}$ & $\begin{array}{l}r=, 0668 \\
p=, 717\end{array}$ & $\begin{array}{l}r=, 2722 \\
p=, 132\end{array}$ & $\begin{array}{l}r=, 0265 \\
p=, 886\end{array}$ & $\begin{array}{l}r=-, 1184 \\
p=, 519\end{array}$ \\
\hline Tempo pós-rádio & $\begin{array}{l}r=-, 0019 \\
p=, 992\end{array}$ & $\begin{array}{l}r=1,0000 \\
p=---\end{array}$ & $\begin{array}{l}r=-, 0295 \\
p=, 873\end{array}$ & $\begin{array}{l}r=, 1916 \\
p=, 293\end{array}$ & $\begin{array}{l}r=, 0743 \\
p=, 686\end{array}$ & $\begin{array}{l}r=-, 0884 \\
p=, 631\end{array}$ & $\begin{array}{l}r=, 1224 \\
p=, 504\end{array}$ \\
\hline OHIP-14 & $\begin{array}{l}\mathrm{r}=, 0668 \\
\mathrm{p}=, 717\end{array}$ & $\begin{array}{l}r=-, 0295 \\
p=, 873\end{array}$ & $\begin{array}{l}r=1,0000 \\
p=---\end{array}$ & $\begin{array}{l}r=, 2909 \\
p=, 106\end{array}$ & $\begin{array}{l}\mathrm{r}=-, 1032 \\
\mathrm{p}=, 574\end{array}$ & $\begin{array}{l}r=-, 0248 \\
p=, 893\end{array}$ & $\begin{array}{l}r=, 0658 \\
p=, 720\end{array}$ \\
\hline Fluxo salivar & $\begin{array}{l}\mathrm{r}=, 0668 \\
\mathrm{p}=, 717\end{array}$ & $\begin{array}{l}r=, 1916 \\
p=, 293\end{array}$ & $\begin{array}{l}r=, 2909 \\
p=, 106\end{array}$ & $\begin{array}{l}r=1,0000 \\
p=---\end{array}$ & $\begin{array}{l}r=-, 2180 \\
p=, 231\end{array}$ & $\begin{array}{l}r=-, 0542 \\
p=, 768\end{array}$ & $\begin{array}{l}r=, 2079 \\
p=, 254\end{array}$ \\
\hline Uso de prótese & $\begin{array}{l}r=, 2722 \\
p=, 132\end{array}$ & $\begin{array}{l}r=-, 0743 \\
p=, 686\end{array}$ & $\begin{array}{l}r=-, 1032 \\
p=, 574\end{array}$ & $\begin{array}{l}r=-, 2180 \\
p=, 231\end{array}$ & $\begin{array}{l}r=1,0000 \\
p=---\end{array}$ & $\begin{array}{l}r=-, 0245 \\
p=, 894\end{array}$ & $\begin{array}{l}r=-, 2980 \\
p=, 098\end{array}$ \\
\hline Necessidade de prótese & $\begin{array}{l}r=, 4734 \\
p=, 006\end{array}$ & $\begin{array}{l}r=, 0675 \\
p=, 714\end{array}$ & $\begin{array}{l}r=-, 1272 \\
p=, 488\end{array}$ & $\begin{array}{l}\mathrm{r}=, 1218 \\
\mathrm{p}=, 507\end{array}$ & $\begin{array}{l}r=, 1077 \\
p=, 557\end{array}$ & $\begin{array}{l}r=-, 1069 \\
p=, 560\end{array}$ & $\begin{array}{l}r=-, 3672 \\
p=, 039\end{array}$ \\
\hline Dose total & $\begin{array}{l}\mathrm{r}=, 0265 \\
\mathrm{p}=, 886\end{array}$ & $\begin{array}{l}r=-, 0884 \\
p=, 631\end{array}$ & $\begin{array}{l}r=-, 0248 \\
p=, 893\end{array}$ & $\begin{array}{l}r=-, 0542 \\
p=, 768\end{array}$ & $\begin{array}{l}r=-, 0245 \\
p=, 894\end{array}$ & $\begin{array}{l}r=1,0000 \\
p=---\end{array}$ & $\begin{array}{l}r=, 0340 \\
p=, 853\end{array}$ \\
\hline
\end{tabular}




\section{DISCUSSÃo}

As variáveis clínicas correlacionadas aos instrumentos de qualidade de vida aplicados foram de importância fundamental no processo de reconhecimento das limitações do paciente entrevistado, assim como todas as propriedades que detectassem sua melhora (BOUCHET et al., 2000). A reabilitação da saúde bucal tem sido atividade frequente no contexto atual (BRASIL, 2012). A análise de correlação demonstrou que quanto maior a idade do paciente, maior a necessidade de prótese $(p=0,006)$. A prevalência do edentulismo aliada à necessidade de prótese afeta a população, principalmente, ao decorrer do envelhecimento pelas características ósseas, sejam estas de reparação mais lenta, rigidez presente, como também as constituintes sociais com parâmetro de que a extração dentária e 0 edentulismo eram processos continuamente feitos. Este cenário apresenta uma perspectiva de falta de medidas preventivas eficazes nesse contexto (SILVA; SOUZA, 2006).

A sialometria em repouso foi um parâmetro conclusivo referente à diminuição do uso e a necessidade de próteses dentárias para os pacientes que apresentaram um fluxo salivar normal, pois em condições normais, este contribui para 0 processo de umedecimento e proteção da mucosa oral, influenciando na diminuição da perda dentária (SILVA,1996). Tal redução decorre de 0 fato da saliva ter propriedades que protegem a superfície, como teor alcalino e, consequentemente, a redução da acidez bucal. A hipossalivação pode predispor o sistema estomatognático a diversas infecções oportunistas (FEIO; SAPETA, 2005). 0 tempo após a radioterapia parece influenciar em alguns casos no aumento do fluxo salivar não estimulado. Este fato correlacionase à presença ou à ausência das glândulas salivares maiores na área irradiada comprometendo ou não a função da saliva no meio bucal (MANDEL, 1987).

0 impacto da saúde bucal na qualidade de vida é fator integrante da saúde geral do indivíduo (PETERSEN, 2003). Alterações bucais que envolvem as funções mastigatórias, estéticas, podem causar desconforto psicológico do paciente, assim como alteração física em virtude de algum processo doloroso presente e assim interferir negativamente em todas as condições diárias (LOCKER, 2000). Em menos de um ano de tratamento não há grande impacto da saúde bucal na qualidade de vida. Seus efeitos podem ser agudos, influenciando tecidos de alta taxa de renovação celular ou tardios, acometendo aqueles de maior especificidade e, assim, agregando maiores fatores negativos na vida deste paciente (ROLIM et al., 2011). 0 impacto produzido no período póstratamento se correlaciona às funções emocionais e cognitivas afetadas no decorrer do tempo que acabam por interferir com as condições locais e sistêmicas do paciente (TOLENTINO et al., 2011).

A escolha dos itens avaliados decorre muito do fato destes parâmetros interferirem diretamente nas relações sociais dos pacientes (OLCKIK et al., 2013). A perda dentária acarreta além de alterações estéticas, problemas na mastigação, deglutição e fala (CUNHA et al., 1999; FAZITO et al., 2004; CALDAS JÚNIOR et al., 2005). Estas mudanças interferem no convívio diário e também são contribuintes para mudanças psicológicas, as quais incluem a necessidade de adaptação do indivíduo com a estabilidade de sua nova prótese (SILVA; GOLDENBERG, 2001; FAJARD0 et al., 2002)

A quantificação da saliva e a análise de sua qualidade auxiliam na percepção de prováveis alterações bucais do paciente, assim como possíveis mudanças sistêmicas presentes. As causas podem ser decorrentes de alguma infecção presente, alterações provenientes do uso de um determinado medicamento, do tipo de tratamento realizado, entre outros. A coleta da saliva contribui, portanto, para monitorar o estado da saúde e da doença de um indivíduo de forma a detectar problemas de forma rápida e eficácia (DAWES, 1987; SLAVKIN, 1999).

0 estudo da qualidade de vida é de íntima relação com todas as alterações físicas e emocionais agravadas não só nos pacientes de câncer de cabeça e pescoço, mas em todos os indivíduos ao decorrer da vida (NERI, 2001). Promover o bem-estar em 
todo o desenvolvimento humano é de fundamental importância neste contexto, sendo considerado um trabalho multidimensional tratar o paciente em todo 0 processo de reabilitação tanto física como psicológica, assim como inclui-lo em uma fase preventiva de possíveis ocorrências (FREIRE, 2003).

Os resultados obtidos sugerem a integração de vários setores profissionais para análise do paciente durante todo o tratamento, assim como em seu período de proservação, visando à adequação de todas suas modificações físicas e emocionais.

\section{CONCLUSÃO}

0 tratamento radioterápico causou diminuição do fluxo salivar em grande parte dos pacientes. 0 edentulismo foi característica marcante no presente estudo produzindo deficiências estéticas e funcionais, e houve correlação entre a maior idade e a necessidade de prótese. A saúde bucal comprometida teve impacto na qualidade de vida de indivíduos irradiados em região de cabeça e pescoço.

\section{REFERÊNCIAS}

ALMEIDA, P. J.; KOWALSKI, L. P. Pilocarpine use to treat xerostomia in patients submitted to radioactive iodine therapy: a pilot study. Braz J Otorhinolaryngol, São Paulo, v. 76, n. 5, p. 659-662, 2010.

AZEVED0, J. S. SBBrasil 2010: uso e necessidade de prótese dentária em idosos. 2014. 59f. Dissertação (Mestrado em Saúde Pública) - Universidade Federal de Pelotas, Pelotas, RS.

BOUCHET, C.; GUILLEMIN, F.; PAUL-DAUPHIN, A.; BRIANÇON, S. Selection of quality-of life measures for a prevention trial: a psychometric analysis. Control Clin Trials, New York, v. 21, n. 1, p. 30-43, 2000.

BRAGA, M. A. Estudo comparativo da ação da pilocarpina e cemivelina na estimulação do fluxo salivar em humanos. 2006. 104f. Monografia (Especialização em Halitose) - Centro de Estudos
Odontológico São Leopoldo Mandic, Campinas, SP.

BRASIL. Ministério da Saúde Conselho Nacional de Saúde. Resolução n 466, 2012. Diretrizes e Normas regulamentadoras de pesquisa envolvendo seres humanos. Ministério da Saúde, Brasília, 2012.

BROWWER, C. L.; STEENBAKKERS, R. J. H. M.; LANGENDIJH, J. Á.; SIJTSEMA, N. M. Identifying patients who may benefit from adaptive radiotherapy: Does the literature on anatomic and dosimetric changes in head and neck organs at risk during radiotherapy provide information to help? Radiother Oncol, Oxford, v. 115, n. 3, p. 285-294, 2015.

CABRERA, M. A. S.; MESAS, A. E.; ROSSATO, L. A.; ANDRADE, S. M. Fluxo salivar e uso de drogas psicoativas em idosos. Rev Assoc Med Bras, São Carlos do Pinhal, v. 53, n. 2, p. 178, 2007.

CALDAS JÚNIOR, A. F. et al. 0 impacto do edentulismo na qualidade de vida de idosos. Rev Ciências Médicas, Campinas, v. 14, n. 3, p. 229-238, 2005.

CHEN, Y. Y. et al. Intensity - modulated radiation therapy reduces radiation induced trismus in patients with nasopharingeal carcinoma. Cancer, Medford, v. 117, n. 13, p. 2910-2916, 2011.

CORREIA, S. F. Reabilitação do doente oncológico da cabeça e pescoço: considerados clínicos e protocolo de atuação. 2013. 57f. Dissertação (Mestrado em Medicina Dentária). Universidade Católica Portuguesa, Viseu, 2013.

CUNHA, C. C.; FELÍCIO, C. M.; BATAGLION, C. Condições miofuncionais orais em usuários de próteses totais. Pró-Fono Ver de Atualização Científica, São Paulo, v. 11, n. 1, p. 21-26, 1999.

DAWES, C. Physiological factors affecting salivary flow rate, oral sugar clearance, and the sensation of dry mouth in man. J Dent Res, Washington, v. 66, p. 648653, 1987.

FAJARDO, R. S.; SUNDEFELD, M. L. M. M.; CAÇÃO, F. M.; et al. Análise das condições funcionais e psicológicas em pacientes edêntulos portadores de próteses totais. Arquivos em Odontologia, Belo Horizonte, v. 38 n. 2, p. 87-94, 2002.

FAZITO, L. T.; PERIM, J. V.; DI NINNO, C. Q. M. S. 
Comparação das queixas alimentares de idosos com e sem prótese dentária. Revista CEFAC, Campinas, v. 6 , n. 2, p. 143-150, 2004.

FEI0, M.; SAPETA, P. Xerostomia em cuidados paliativos. Acta Med Port, v. 18, p. 459-466, 2005.

FOGER D. Capacidade funcional e saúde bucal relacionada à qualidade de vida de idosos. 2015. 111f. Dissertação (Mestrado em ciências). Faculdade de Odontologia de Bauru - Universidade de São Paulo, Bauru, SP.

FREIRE, S. A. Educação permanente e qualidade de vida na velhice: meta e desafio na atualidade. In:CONGRESSO PAULISTA DE GERIATRIA E GERONTOLOGIA, 3., 2003, Santos. Anais... Santos: SBGG, 2003.

HADDAD, R. I.; SHIN, D. M. Recent advances in head and neck cancer. N Eng J Med, Massachusetts, v. 359, n. 11, p. 1143-1154, 2008.

HUTCHESON, K. A. et al. Late dysphagia after radiotherapy based treatment of head and neck cancer. Cancer, Medford, v. 118, n. 26, p. 5793-5799, 2012.

JEMAL, A.; SIEGEL, R.; WARD, E.; HAO, Y.; XU, J.; THUN, M. J. Cancer statistics. CA Cancer J Clin, Atlanta, v. 59, n. 4, p. 225-249, 2009.

LOCKER, D. Deprivation and oral health: a review. Community Dent Oral epidemiol, Medford, v. 28, p.161-169, 2000.

MANDEL, I. D. The functions of saliva. J Dent Res, Washington, v. 66, p. 623-627, 1987.

MARQUES, N. C. C. Osteorradionecrose dos maxilares. 2015. 80f. Dissertação (Mestrado em medicina dentária). Faculdade de Ciências Universidade Fernando Pessoa, Porto, Portugal.

NERI, A.L. Velhice e qualidade de vida na mulher. In: NERI, A.L. Desenvolvimento e envelhecimento: perspectivas biológicas, psicológicas e sociológicas. Campinas, SP: Papirus, 2001.p.161-200.

OLCHIK, M. R.; AYRES, A., PRESOTTO, M.; BALTEZAN, R. L.; GONÇALVES, A. K. 0 impacto do uso de prótese dentária na qualidade de vida de adultos e idosos. Revista Kairós Gerontologia, São Paulo, v. 16, n. 5, p. 107-121, 2013.
PEDRAZAS, C. H. S.; AZEVEDO, M. N. L.; TORRES, S. R. Manejo do paciente com hipossalivação. Revista PerioNews, São Paulo, v. 1, n. 4, p. 369, 2007.

PETERSEN, P. E. The world oral health report 2003: continuous improvement of oral in the $21^{\text {st }}$ century - the approach of the WHO Global Oral Health Programme. Community Dent Oral Epidemiol, Medford, v. 31, n. 1, p. 3-33, 2003.

ROLIM, A. E. H.; COSTA, L. J.; RAMALHO, L. M. P. Repercussões da radioterapia na região orofacial e seu tratamento. Radiol Bras, São Paulo, v. 44, n. 6, p. 388395, 2011.

SILVA, E. F. A.; SOUSA, M. L. R. Autopercepção da saúde bucal e satisfação com a vida em mulheres idosas usuárias de prótese total. Rev Odontol Univ Cid São Paulo, São Paulo, v. 18, n. 1, p. 61-65, 2006.

SILVA, L. G.; GOLDENBERG, M. A mastigação no processo de envelhecimento. Revista CEFAC, São Paulo, v. 3, p. 27-35, 2001.

SILVA, R. C. Saliva: muitos cirurgiões-dentistas não Ihe dão a devida atenção. Odontólogo Moderno, São Paulo, v. 23, n. 1, p. 10-13,1996.

SLAVKIN, H. C. Streptococcus mutans, early childhood caries and new opportunities. J Am Dent Assoc, New York, v.130, n.12, p.1787-1792, 1999.

TARZIA, 0. Halitose: um desafio que tem cura. Rio de Janeiro: EPUB, 2004.

THARIAT, J. et al. Oral complications of câncer and câncer therapy: from câncer treatment to survivorship. Ca Câncer J Clin, Atlanta, v. 62, p. 400-422, 2012.

TOLENTINO, E. S.; CENTURION, B. S.; FERREIRA, L. H. C.; SOUZA, A. P.; DAMANTE, J. H.; RUBIRA-BULLEN, I. R. F. Oral adverse effects of head and neck radiotherapy: literature review and suggestion of a clinical oral care guideline for irradiated patients. J Appl Oral Sci, Bauru, v. 19, n. 5, p. 448-454, 2011.

Recebido em: 30 de agosto de 2017 Aceito em: 03 de outubro de 2017 